\title{
Balanced Scorecard para extraer conocimiento de la tecnología
}

Fernando Méndez.

\section{García}

Escuela Superior de Ingeniería Mecánica y Eléctrica, Unidad Culhuacán, Instituto Politécnico Nacional

fdomendezg@yahoo.com.mx

\section{Octavio Ernesto}

Dominguez Sosa

Escuela Superior de Ingeniería Mecánica y Eléctrica, Unidad Culhuacán, Instituto Politécnico Nacional oedominguez@segob.gob.mx.

\section{Fernando Elí Ortiz}

Hernández.

Escuela Superior de Ingeniería Mecánica y Eléctrica, Unidad Culhuacán, Instituto Politécnico Nacional

eliortiz@mexico.com

\section{Resumen}

En la globalización, la innovación tecnológica (IT) y el capital intelectual (CI) son fuerzas que ayudan a las instituciones a crecer y a alcanzar sus objetivos. Para lograrlos, se han desarrollado herramientas que ayudan a las organizaciones a identificar los puntos medulares y sus relaciones para traducir las acciones en resultados concretos; uno de esos instrumentos es el Balanced Scorecard (BSC), el cual identifica los elementos clave de la compañía para manejarlos de manera sistemática y estructurada bajo relaciones de causa-efecto. En este trabajo se adapta la metodología del BSC a un proyecto de innovación tecnológica de una institución del sector público mexicano encargada de aplicar la Ley Federal de Radio y Televisión con el propósito de extraer el CI y mayores beneficios de la tecnología adquirida. De la adaptación, surge el tablero de capacidades tecnológicas (Тст) y la cadena de valor tecnológica, cuyos eslabones principales son la IT y el cI; las diferencias técnicas entre cada uno de ellos impactan los objetivos organizacionales. Las variaciones entre los eslabones constituyen áreas de oportunidad donde aplica el тст con el fin de incrementar el conocimiento, las capacidades de la organización y, en consecuencia, los beneficios tangibles e intangibles de la tecnología.

Palabras clave: innovación tecnológica, capital intelectual, cadena de valor tecnológica, tablero de capacidades tecnológicas, cuadro de mando integral. 


\title{
Balanced scorecard to extract knowledge out of technology
}

\begin{abstract}
In globalization, technological innovation and intellectual capital are forces that help institutions to grow and reach their goals. To achieve this, tools have been developed to assist organizations in identifying core points and their relationships to translate actions into concrete results. One such tool is the Balanced Scorecard (BSC), which identifies the key elements of the company in order to manage them in a systematic and structured way under cause-effect relationships. This paper adapts the BSC methodology to a technological innovation project of a Mexican public sector institution, responsible for implementing the Federal Radio and Television laws, in order to extract greater benefits out of intellectual capital from the acquired technology. As a result of this adaptation, there arises a Technological Capabilities Panel and the Technological Value Chain, whose major links are technological innovation and intellectual capital, with the technical differences between each of them impacting organizational objectives. Variations between the links constitute areas of opportunity where the Technological Capabilities Panel is applied in order to increase knowledge, organizational capabilities and, consequentially, the tangible and intangible benefits of technology.
\end{abstract}

Keywords: Technological Innovation, Intellectual Capital, Technological Value Chain, Board of Technological Capabilities, Balanced Scorecard.

\section{Introducción}

En la economía actual hay instrumentos que impulsan a las empresas e instituciones a crecer y a alcanzar sus metas. Dentro de la economía del conocimiento, la innovación tecnológica (IT) y el capital intelectual (CI) ayudan a las organizaciones a concretar su misión y visión si trabajan de manera coordinada y orientada.

Identificar cómo se relacionan la IT (o tecnología) con el cI puede producir beneficios económicos y de otra naturaleza, como es el conocimiento para mejorar el desempeño, para la creación de ventaja competitiva y de competencia medular. ${ }^{1}$

\footnotetext{
${ }^{1}$ Ventaja competitiva, según Porter (1982), es la capacidad de una empresa para defender su posición dentro de la industria y generar retornos de la inversión. La ventaja competitiva se alcanza al producir a más bajo costo, fabricar cosas diferentes, vender productos antes que la competencia; es decir, adelantarse a las necesidades del cliente. De acuerdo con Prahalad \& Hamel (1990) la competencia medular es la capacidad de producir un bien especializado con aplicaciones en varias industrias.
} 
Según la Organización para la Cooperación y el Desarrollo Económico (OCDE) a la tecnología se le puede extraer conocimiento (OCDE, 1997); sin embargo, existen organismos públicos que carecen de herramientas para obtener el máximo beneficio de la tecnología adquirida, lo que constituye un serio problema para la creación de nuevas capacidades. La tecnología puede contribuir con la organización para incrementar sus ingresos, su oferta de productos, fortalecer su posición en el mercado y ejercer control sobre la industria y, desde luego, para ampliar y fortalecer sus conocimientos, renovar sus procesos y el tipo de organización, pero debe ser manejada con los criterios técnicos y administrativos adecuados. Nonaka y Takeuchi (1995: 84) ponen de ejemplo a la empresa NEC, cuando la firma consideró a la tecnología como un sistema de conocimiento formulando programas de capacidades medulares en sus laboratorios centrales de investigación.

La falta de instrumentos tecnológico-administrativos representa un problema para las instituciones, pues les impide observar los puntos importantes donde convergen la tecnología y el conocimiento - como una forma de analizar las relaciones y los productos que generan- para que a través de acciones concretas sobre la tecnología se logre mayor formación de cr en una situación de causa-efecto. Esto lo han advertido investigadores como Martín de Castro y López (2008) cuando argumentan que existen pocos esfuerzos teóricos y prácticos para adaptar los modelos del cr en otros campos económicos y sociales; en este caso, en una institución pública mexicana, fiscalizadora y reguladora de los tiempos oficiales en radio y televisión (TV) y de los contenidos difundidos a través de esos medios de comunicación, que ha introducido recientemente nuevas tecnologías para simplificar sus procesos. ${ }^{2}$ No obstante que la propuesta de un tablero de capacidades tecnológicas (ТСТ) se deriva de un problema en una dependencia del Gobierno mexicano, es un argumento válido también para empresas privadas, pues cualquier tipo de sociedad depende y compra tecnología; es por esto que en este trabajo se hace referencia a institución, organización, empresa, compañía y firma de manera indistinta. Todos pueden extraer conocimiento de los equipos adquiridos siempre y cuando cuenten con las herramientas necesarias.

${ }^{2}$ Los tiempos oficiales se dividen en tiempos fiscales y tiempos de estado, los primeros son los impuestos en tiempo de transmisión que pagan los permisionarios y concesionarios de radio y TV al gobierno por el uso del espectro radio eléctrico, el cual consiste en 18 minutos diarios para las televisoras y 35 en las radiodifusoras (DOF, 2002). Los segundos son, según el artículo 15 del Reglamento de la Ley Federal de Radio y Televisión, la obligación de las televisoras y radiodifusoras de transmitir gratuitamente en su programación diaria, 30 minutos continuos o discontinuos sobre temas educativos, culturales, sociales, políticos, deportivos y otros de interés general (DOF, 2002a). Las funciones de regulación de la institución de referencia se contemplan dentro de los artículos 1 al 10 de la Ley Federal de Radio y Televisión (DOF, 2009). 
La falta de dichos instrumentos motiva la propuesta de un instrumento que permita a los directivos de la organización identificar y administrar los elementos del CI dentro de los proyectos tecnológicos —en este caso en el Sistema de Monitoreo y Distribución de Señales de Radio y TV (SIMODI) — con el fin de dirigirlos en la búsqueda de la misión y visión desde el enfoque del Balanced Scorecard (BSC). Así la cuestión por investigar es ¿cómo debería funcionar un тСт que permita a la organización incrementar el cr a partir de la tecnología introducida?, pues hasta el momento las IT implementadas no le han permitido elevar el conocimiento y las capacidades organizacionales. Para responder a la pregunta se reseña, en el marco teórico, la literatura existente sobre el tema, se establece al BSC como el método más común para formar CI en las empresas para, posteriormente, aplicar la citada metodología en el sIMODI, obteniendo como resultado la cadena de valor tecnológico (CVT) y el тст. Por último, se ofrecen a manera de conclusión, consideraciones generales sobre el TCT y de la integración conceptual de la IT y el CI.

\section{Marco teórico}

De acuerdo con Schumpeter (1944) innovar es introducir un nuevo bien o servicio, un nuevo método o proceso de producción, un nuevo mercado, una nueva fuente de materias primas, de manufacturas o una nueva organización. El término innovación se utiliza para designar a cualquier "cosa nueva" en el área de la ciencia, la tecnología o el arte. Cuando se requiera ser más preciso la innovación puede ir seguida de un adjetivo adecuado: "innovación científica", "innovación técnica", "innovación en la organización", "innovación de procesos", o en algo aún más preciso como: "innovación tecnológica en sistemas informáticos" (Ruttan, 1979).

Freeman (1982) define a la innovación como la nueva tecnología que ofrece nuevos productos y servicios a los clientes. Van de Ven (1986) la precisa como el desarrollo e implementación de nuevas ideas de un contexto a otro. La innovación es un proceso de creación de nuevas cosas en la organización formadoras de valor (Khalil, 2000; Narvekar y Karuna, 2006).

Con respecto a la tecnología, Gaynor (1999) la define como el conjunto de conocimientos e instrumentos necesarios para el diseño de productos y/o procesos en la búsqueda de nuevo conocimiento. Cuando la tecnología interviene en la venta de nuevos productos, procesos o servicios al mercado se convierte en investigación tecnológica (IT) (Betz, 1993); o bien, cuando los productos o procesos son sustancialmente mejorados (OCDE, 1997). La IT conjuga una oportunidad con una necesi- 
dad de mercado a través del desarrollo o mejora de productos, procesos, servicios y materiales, que debe ser significativamente novedosa (Conacyt, 2007). Sin duda, la IT es la mejora en los conocimientos, los instrumentos y los procesos que hacen posible elaborar y entregar a los clientes y/o usuarios, los productos y servicios en mejores condiciones que los existentes, lo cual produce ganancias tangibles e intangibles para las instituciones.

En cuanto al término capital intelectual (CI), fue Tomas Stewart quien acuñó el vocablo en referencia a los estudios de creación de valor de John Kenneth Galbraith y Michael Kalecki y lo definió como la suma de todo lo que da competitividad en el mercado. Lo clasificó en capital estructural, capital cliente y capital humano (Sveiby, 1998). En cambio para Annie Brooking (1997) el cI son todas las aportaciones no materiales que conforman el principal activo para las empresas. El cr es conocimiento creador de valor (Edvinsson y Malone, 1997; Sullivan, 2001).

En el nivel individual, el CI incluye todos los conocimientos de los empleados; es la fuerza cerebral colectiva que proporciona ventaja competitiva a la empresa (Stewart, 1998); el capital está en las cabezas y no en las cuentas bancarias (Deutschland, 2009). Por su parte, el nivel organizacional comprende la experiencia y las destrezas profesionales de las personas, la tecnología organizacional, las relaciones con clientes (Milena, 2005), la información y la propiedad intelectual (Stewart, 1998), así como la clientela, el nombre, la razón social, la localización, la cuota de mercado, el nivel de competencia, los canales comerciales y otros de naturaleza análoga que impliquen valor para la empresa (García, 1996). Gómez (2005) introduce al cr el liderazgo tecnológico, las marcas comerciales y los gastos capaces de adquirir mayor valor futuro. Fiona Murray (2004) sostiene que capital es conocimiento capturado en patentes y publicaciones.

Para que el CI genere valor requiere de una estrategia directiva o de management. Sveiby (1998) considera que el cr y el manejo del conocimiento son ramas del mismo árbol; por lo tanto, el cr es todo aquel conocimiento tácito o explícito capaz de crear valor a partir de una estrategia; además de que otorga una ventaja competitiva o medular en cualquier mercado, sea empresarial, social, financiero u otro.

Por lo que respecta a la IT, el conocimiento vende nuevos productos al mercado en mejores condiciones y con el CI construye ventaja competitiva y competencia medular. La primera se sintetiza en ser el mejor del mercado, poseer o hacer algo único (Méndez y Ortiz, 2007); en tanto que la segunda es producir un bien líder en 
varias industrias (Prahalad y Hamel, 1990). Todo como resultado del conocimiento y plataforma para su reproducción.

Tayles, Pike y Sofian (2007) identificaron que los directivos emplean indistintamente los términos intangibles, conocimiento y cI, pero siempre refiriéndose a una fuente de beneficios futuros sin sustancia física (Lev, 2003). El CI es sinónimo de conocimiento organizacional (Narvekar y Karuna, 2006).

Por su parte, Karl Erik Sveiby (Tayles et al., 2007) fue el primero en identificar el CI en modelos empresariales; lo clasificó en: 1) estructura externa, que son aquellas relaciones exteriores y los símbolos que identifican a la empresa, como marcas e imagen; 2) estructura interna, es el conocimiento codificado y estructurado y el personal encargado de su mantenimiento; y 3 ) competencias, las cuales pueden ser individuales y grupales que producen y dan soluciones (Sveiby, 1996). Este autor denominó a su modelo monitor de activos intangibles (MAI); posteriormente, han surgido otros modelos que clasifican el ci de manera distinta, como se muestra en el cuadro 1.

Como se observa en ese cuadro, el objetivo de los modelos del CI es operativo, pues se ubica en los procesos y la extracción de valor; al aspecto financiero, por una parte, al responsabilizarlo de las variaciones en los diferentes tipos de valor; por otra, administrativo, al insertarlo en operaciones estratégicas, gestión y elaboración de reportes de resultados, incluido el de cultura organizacional. 


\section{Cuadro 1}

\section{Modelos del capital intelectual}

\begin{tabular}{|c|c|c|c|c|c|c|c|}
\hline Modelo & Autor & Año & Objetivo & $\begin{array}{l}\text { Empresa } \\
\text { donde } \\
\text { aplicó }\end{array}$ & \multicolumn{3}{|c|}{ Clasificación del capital intelectual } \\
\hline $\begin{array}{l}\text { Monitor } \\
\text { de Activos } \\
\text { Intangibles }\end{array}$ & $\begin{array}{l}\text { Karl Erik } \\
\text { Sveiby }\end{array}$ & 1986 & $\begin{array}{l}\text { Explicar } \\
\text { el capital } \\
\text { intelectual } \\
\text { en los } \\
\text { procesos }\end{array}$ & Ninguna & $\begin{array}{l}\text { Estructura } \\
\text { interna }\end{array}$ & $\begin{array}{l}\text { Estructura } \\
\text { externa }\end{array}$ & $\begin{array}{l}\text { Competen- } \\
\text { cias }\end{array}$ \\
\hline $\begin{array}{l}\text { Navegador } \\
\text { de Skandia }\end{array}$ & $\begin{array}{l}\text { Leif } \\
\text { Edvinsson } \\
\text { y } \\
\text { M. } \\
\text { Malone }\end{array}$ & 1991 & $\begin{array}{l}\text { Identificar las } \\
\text { diferencias } \\
\text { entre el valor } \\
\text { contable } \\
\text { y el valor } \\
\text { financiero }\end{array}$ & Skandia & $\begin{array}{l}\text { Capital } \\
\text { estructural }\end{array}$ & $\begin{array}{l}\text { Capital } \\
\text { clientes }\end{array}$ & $\begin{array}{l}\text { Capital } \\
\text { humano }\end{array}$ \\
\hline $\begin{array}{l}\text { Balanced } \\
\text { Scorecard }\end{array}$ & $\begin{array}{l}\text { Kaplan y } \\
\text { Norton }\end{array}$ & 1992 & $\begin{array}{l}\text { Traduce las } \\
\text { estrategias en } \\
\text { mapas y } \\
\text { métricas } \\
\text { asociadas a } \\
\text { proyectos } \\
\text { clave }\end{array}$ & Ninguna & $\begin{array}{l}\text { Perspectiva } \\
\text { interna y } \\
\text { financiera de } \\
\text { la empresa }\end{array}$ & $\begin{array}{l}\text { Perspectiva } \\
\text { del } \\
\text { cliente }\end{array}$ & $\begin{array}{l}\text { Perspectiva } \\
\text { de } \\
\text { innovación y } \\
\text { aprendizaje }\end{array}$ \\
\hline $\begin{array}{l}\text { Dow } \\
\text { Chemical }\end{array}$ & $\begin{array}{l}\text { Gordon } \\
\text { Petrash }\end{array}$ & 1995 & $\begin{array}{l}\text { Gestión } \\
\text { de activos } \\
\text { intelectuales }\end{array}$ & $\begin{array}{l}\text { Dow } \\
\text { Chemical }\end{array}$ & $\begin{array}{l}\text { Capital } \\
\text { organización }\end{array}$ & $\begin{array}{l}\text { Capital } \\
\text { cliente }\end{array}$ & $\begin{array}{l}\text { Capital } \\
\text { humano }\end{array}$ \\
\hline $\begin{array}{l}\text { Celemi } \\
\text { Monitor }\end{array}$ & $\begin{array}{l}\text { Empresa } \\
\text { Celemi }\end{array}$ & 1995 & $\begin{array}{l}\text { Reportar } \\
\text { resultados }\end{array}$ & Celemi & $\begin{array}{l}\text { Nuestra } \\
\text { organización }\end{array}$ & $\begin{array}{l}\text { Nuestros } \\
\text { clientes }\end{array}$ & $\begin{array}{l}\text { Nuestra } \\
\text { gente }\end{array}$ \\
\hline $\begin{array}{l}\text { Canadian } \\
\text { Imperial } \\
\text { Bank }\end{array}$ & $\begin{array}{l}\text { Hubert } \\
\text { Saint- } \\
\text { Honge }\end{array}$ & 1996 & $\begin{array}{l}\text { Explicar } \\
\text { la cultura } \\
\text { organizacional }\end{array}$ & $\begin{array}{l}\text { Canadian } \\
\text { Imperial } \\
\text { Bank }\end{array}$ & $\begin{array}{l}\text { Capital } \\
\text { estructural }\end{array}$ & $\begin{array}{l}\text { Capital } \\
\text { clientes }\end{array}$ & $\begin{array}{l}\text { Capital } \\
\text { humano }\end{array}$ \\
\hline $\begin{array}{l}\text { Empresa de } \\
\text { Conoci- } \\
\text { miento }\end{array}$ & $\begin{array}{l}\text { Patrick } \\
\text { Sullivan }\end{array}$ & 1996 & $\begin{array}{l}\text { Identificar } \\
\text { fuentes de } \\
\text { valor y su } \\
\text { extracción }\end{array}$ & $\begin{array}{l}\text { Xerox y } \\
\text { Hewlett } \\
\text { Packard }\end{array}$ & $\begin{array}{l}\text { Capital } \\
\text { estructural }\end{array}$ & $\begin{array}{l}\text { Activos } \\
\text { empresariales } \\
\text { complemen- } \\
\text { tarios }\end{array}$ & $\begin{array}{l}\text { Capital } \\
\text { humano y } \\
\text { activos } \\
\text { intelectuales }\end{array}$ \\
\hline $\begin{array}{l}\text { Technology } \\
\text { Broker }\end{array}$ & $\begin{array}{l}\text { Annie } \\
\text { Brooking }\end{array}$ & 1996 & $\begin{array}{l}\text { Metodología } \\
\text { para identificar } \\
\text { el valor de } \\
\text { mercado de } \\
\text { una empresa }\end{array}$ & Ninguna & $\begin{array}{l}\text { Activos de } \\
\text { infraestructura } \\
\text { y de propiedad } \\
\text { intelectual }\end{array}$ & $\begin{array}{l}\text { Activos de } \\
\text { mercado }\end{array}$ & $\begin{array}{l}\text { Activos } \\
\text { humanos }\end{array}$ \\
\hline Intelect & $\begin{array}{l}\text { Euro- } \\
\text { forum }\end{array}$ & 1998 & $\begin{array}{l}\text { Ofrecer } \\
\text { información } \\
\text { para la gestión }\end{array}$ & Ninguna & $\begin{array}{l}\text { Capital } \\
\text { estructural }\end{array}$ & $\begin{array}{l}\text { Capital } \\
\text { relacional }\end{array}$ & $\begin{array}{l}\text { Capital } \\
\text { humano }\end{array}$ \\
\hline
\end{tabular}

La clasificación de ci más utilizada es la de capital humano, capital relacional y capital estructural del modelo Intelec, que ofrece información para la gestión, describe los elementos del CI, y los creadores de valor en las organizaciones. Según esta 
clasificación, el capital humano es todo tipo de conocimiento con potencial para replicarlo y multiplicarlo; el capital relacional es el valor de las relaciones con el exterior, precursoras del éxito y generadoras de oportunidades futuras; y el capital estructural es el conocimiento explícito y organizado que reditúa beneficios para la empresa (Gestión, 2006). Narvekar y Karuna (2006) emplearon dicha clasificación para elaborar un marco teórico de los procesos de IT; Johanson, Koga, Skoog y Henningsson (2006) la utilizaron para analizar el cr del gobierno japonés; Tayles et al. (2007) la usaron para medir la percepción de los managers en el desempeño corporativo; Martín de Castro y López (2008) la identificaron en firmas españolas de alta tecnología; sin embargo, ninguno desarrolló modelos gráficos.

Un modelo, de acuerdo con Varian (1999:2), es una "representación simplificada de la realidad"; se emplea como un formato o sistema de registro para la toma de decisiones; el más popular es el Balanced Scorecard de Kaplan y Norton (Petty, Ricceri \& Guthrie, 2008), que se muestra a continuación.

Figura 1

Balanced Scorecard

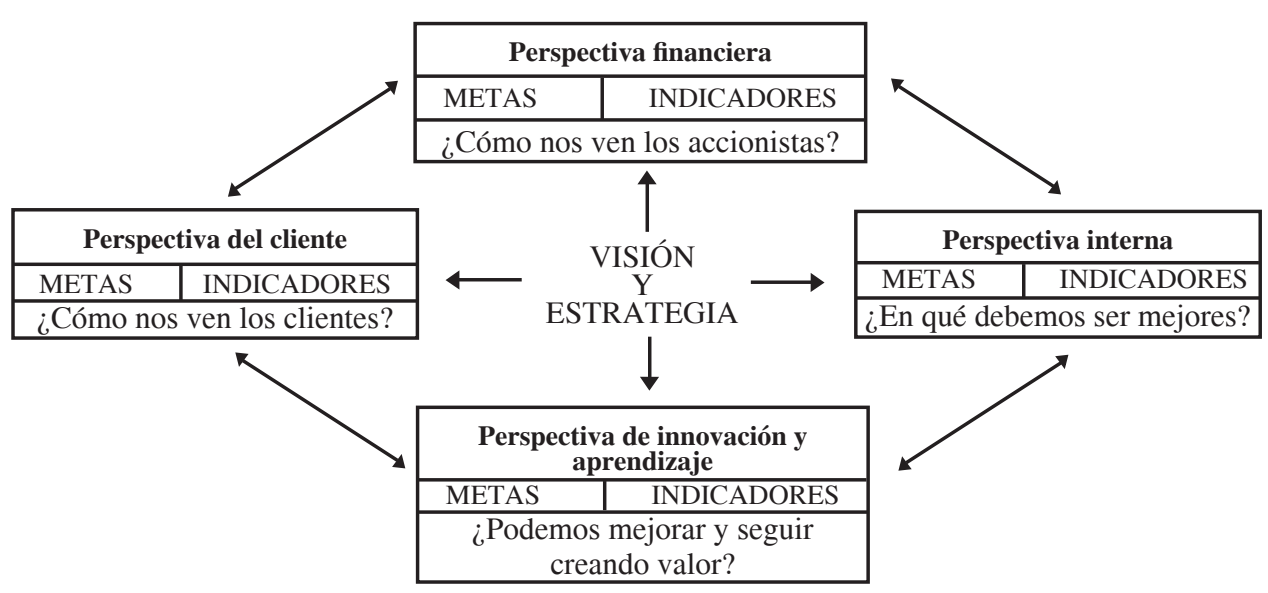

Fuente: Kaplan y Norton (1992).

El primer intento por crear un tablero de indicadores fue el Tableau du Bord francés, el cual generó indicadores financieros y no financieros, mismos que - a criterio de los directivos- eran los más importantes de la realidad organizacional porque reduce los errores humanos. Fue hasta 1992 cuando Robert S. Kaplan y David 
Norton dieron a conocer el BSC actual en su artículo "The Balanced Scorecard: Measures that Drive Performance"; en 1996 lo afinaron en su libro The Balanced Scorecard: Traslading Strategy into Action (KPMG, 2005). En el siguiente cuadro se muestran sus objetivos y ventajas.

\section{Cuadro 2 \\ Objetivos y ventajas del BSC}

\begin{tabular}{|c|c|}
\hline \multicolumn{2}{|c|}{ Balanced Scorecard } \\
\hline Objetivos & Ventajas \\
\hline $\begin{array}{l}\text { - Mantener un enfoque estratégico y poder } \\
\text { evaluar la gestión de las estrategias. } \\
\text { - Obtener claridad y consenso alrededor de } \\
\text { las estrategias. } \\
\text { - Desarrollar liderazgo entre los miembros } \\
\text { de la organización. } \\
\text { - Educar a la organización. } \\
\text { - Fijar metas estratégicas claras. } \\
\text { - Alinear los programas y las inversiones. } \\
\text { - Mejorar el sistema de indicadores actuales. }\end{array}$ & $\begin{array}{l}\text { - Reduce al mínimo la sobrecarga de información. } \\
\text { - Reúne en un solo informe elementos claves de la } \\
\text { organización. } \\
\text { - Previene subestimar aspectos que tradi-cionalmente } \\
\text { no son claves. } \\
\text { - Correlaciona varios aspectos importantes y permite } \\
\text { visualizar efectos. } \\
\text { - Agrupa varias dimensiones y perspectivas en un } \\
\text { solo tablero. } \\
\text { - Permite tener una comprensión global de la } \\
\text { organización. }\end{array}$ \\
\hline
\end{tabular}

Fuente: KPMG (2005).

El BSC es la metodología más utilizada para desarrollar el CI en las organizaciones; más del 50\% de las empresas listadas en la revista Fortune lo utilizan (Weinstein y Bukovinsky, 2009); más de la mitad de las empresas de Estados Unidos (EE.UU) lo emplean (Pangakar y Kirkwood, 2008). En México, la firma de consultoría Gestión de Negocios vende la aplicación y el software; sin embargo, como sostienen Weinstein y Bukovinsky (2009), todavía existen campos donde faltan sistemas de medición; estos autores aplicaron el BSC a una compañía teatral de Boston, EE.UU; Spivey, Munson, Flannery y Tsai (2009) formularon un BSC para alianzas tecnológicas entre productores y proveedores de distintos países.

Cada autor o empresa diseña el BSC según sus necesidades y maneja las perspectivas de acuerdo con ello, pues son flexibles. Lipe y Salterio (2000) distinguen entre las perspectivas, medidas únicas y medidas comunes, las primeras valoran el desempeño de una unidad de negocio y las segundas son parámetros de resultados financieros corporativos. Dado que estas últimas son más fáciles de medir en términos económicos se consideran como la principal perspectiva cuando se trata de 
incrementar los recursos y con ese propósito se subordinan las demás perspectivas (Pangakar y Kirkwood, 2008), tal como lo hizo la compañía teatral de Boston que identificó medidas únicas vinculadas entre todas las perspectivas y después elaboró métricas para monitorear el desempeño organizacional. Banker, Chang y Pizzini (2004) señalan que cuando las medidas únicas están vinculadas estratégicamente con los objetivos son más importantes que las financieras a la hora de tomar decisiones. Es importante aclarar que con el Bsc la compañía teatral incrementó sus ingresos en seis millones de dólares en 2005 y ganó un patrocinio anual de \$500,000 USD de la fundación Wallace (Weinstein y Bukovinsky, 2009).

Así, el BSC o cuadro de mando integral (CMI), como se traduce al español, puede manejar las perspectivas como se requieran, siempre incluyendo un conjunto de métricas para el monitoreo de los objetivos estratégicos, vinculados entre sí relativos al mercado, clientes, procesos internos, CI, aprendizaje, responsabilidad social, medioambiente e infraestructura, entre otros (KPMG, 2005).

El BSC puede adoptar una presentación distinta a la tradicional; por ejemplo, el BSC para alianzas estratégicas de Spivey et al. (2009) tiene cuatro dimensiones diferentes a las de Kaplan y Norton (1992): gestión de activos, acciones de valor, equilibrio empresarial y gestión del conocimiento con metas individuales en cada una para capturar la complejidad de una alianza estratégica en tecnología. El propósito de este CMI fue diseñar una cadena para asegurar los recursos necesarios que genere certidumbre presente y futura. Evalúa la capacidad de los proveedores en la creación de valor a través de la transferencia tecnológica; mediante alianzas se les traspasa responsabilidad más allá de suministrar materiales, equipos y experiencia para crear y desarrollar un portafolio de productos. Los estándares de entendimiento aseguran que cada proveedor conozca anticipadamente lo que se espera de él, tanto en los suministros como en el desarrollo de productos o servicios; funciona como capital relacional generando oportunidades futuras. Esto incrementa los ingresos y reduce costos; asegura un desempeño óptimo de los participantes en la cadena de producción. Las medidas únicas de Lipe y Salterio (2000) llevan a concretar las medidas financieras.

Se genera mayor valor cuando se administra la información de todas las actividades y de los agentes que intervienen en los procesos (Spivey et al., 2009); por ello, Pangakar y Kirkwood (2008) alinean la estrategia y los objetivos de negocios con el aprendizaje y la misión en el BSC. Philip (2007) afirma que se tienen mayores resultados cuando la planeación, la estrategia, los procesos, las tecnologías y las 
acciones están definidas y alineadas; cuando todo se vincula con el modelo de negocios, las medidas no financieras se vuelven más importantes a la hora de evaluar el BSC (Banker et al., 2004).

\section{Desarrollo y aplicación de un BSC tipo TCT}

Desde estos principios se propone un Balanced Scorecard para vincular el capital intelectual con la innovación tecnológica y monitorear el flujo técnico entre las perspectivas - en una institución donde ambas variables trabajan de manera desalineada; es decir, donde no se relacionan las capacidades tecnológicas de los equipos, de los operarios y de las áreas para el desarrollo de sus procesos medulares- como una forma para mejorar su desempeño. Sería un instrumento adecuado para ampliar sus capacidades y cumplir su misión y visión ante nuevos participantes, por ejemplo, de la industria de radio y TV. Frente a cambios en las trayectorias tecnológicas de los agentes del mercado y ante la aparición de nuevas tecnologías para difundir señales de radio y TV como son Digital Video Broadcasting Handheld (DVB-H), la TV de Protocolo de Internet (IPTV), CPE (Customer Premise Equipment), VistaMAX, ZigBee, Base Stations, antenas omnidireccionales y sectoriales, Power Over Ethernet, QoS (Quality of Service), administración del espectro, triangulación, protocolos en tiempo real, Wi Fi puro (Arvizu, 2007), la institución corre el riesgo de quedar imposibilitada de hacer cumplir la Ley Federal de Radio y TV y de sus respectivos reglamentos en esos nuevos medios de comunicación, así como de regular, vigilar y supervisar sus transmisiones y la vigencia de los tiempos oficiales.

Entonces el BSC propuesto identifica los puntos importantes en la corriente tecnológica del simodi para ampliar sus capacidades, asignando medidas únicas de desempeño a las actividades sustantivas de cada perspectiva para conocer su nivel de funcionamiento y contribución con los procesos medulares y en la ampliación de mercado. ${ }^{3}$ Este BSC se formula sobre un proyecto de IT con funcionamiento transversal en la organización que influye en sus resultados, de allí que se elabore a partir de medidas únicas sobre las actividades sustantivas de los equipos, los operarios y las áreas usuarias de la tecnología, para llegar a medidas financieras corporativas, de fiscalización y aplicación de infracciones.

\footnotetext{
${ }^{3}$ Las actividades sustantivas son las tareas que sostienen a los productos y servicios tecnológicos. Los procesos medulares son los flujos tecnológicos que se inician a partir de esas actividades y que recorren las perspectivas del BSC.
} 
Las quejas de los directores de área cuando dicen que la IT no funciona, y al tratar de identificar las razones por las cuáles no se han generado nuevas capacidades, llevó a la administración de la dependencia a solicitar a los autores, indagar las causas por las cuales la tecnología no ha beneficiado a la institución de manera amplia. Un primer resultado de la investigación con tres de las principales áreas usuarias fue que ni ellos, ni la administración de la organización cuentan con un estándar de entendimiento tecnológico. Mediante oficios se les preguntó a los directores de área sobre las funciones y procesos medulares del sIMODI y respondieron cosas totalmente distintas a las esperadas, lo cual evidencia el desconocimiento de las prestaciones de los equipos y la forma en cómo contribuyen a enriquecer sus procesos; en consecuencia, el conocimiento individual y organizacional es limitado. ${ }^{4}$ El BSC destaca las conexiones tecnológicas entre los aparatos, los operadores, los departamentos y las direcciones de área involucradas con la IT, organizados cada uno bajo diferente perspectiva. Por lo tanto, la respuesta a la pregunta de investigación es que la institución requiere un BSC tipo dashboard, el cual se denomina tablero de capacidades tecnológicas. Éste reporta los puntos clave del flujo tecnológico entre las perspectivas, detecta las capacidades sustantivas ofrecidas por los equipos y del personal que los maneja dentro de los procesos medulares de los departamentos y las direcciones de área. Su funcionamiento es como el tablero del automóvil porque muestra las metas e indicadores de funcionamiento de las partes importantes. Un dashboard contiene controles básicos que proporcionan retroalimentación (KPMG, 2005) para corregir errores en los equipos o en el personal dentro de sus actividades sustantivas y los procesos medulares, como lo señalan las experiencias previas de implementación del BSC. El TCT parte de relaciones lógicas y no pretende una racionalidad financiera como sugiere Norreklit (2000), pues se trata de una entidad pública con fines sociales; aquí el BSC es de control y ejecución. El тст toma elementos de la tecnología como generadores de valor interno y externo - forma conocimientos y capacidades nuevas, así como amplía la cobertura de mercado- para evaluarlos y mejorarlos a través de la retroalimentación.

El тст opera dentro del simodi y parte de lo particular a lo general. Como el interés primario es incrementar el CI a partir de la tecnología introducida, la principal perspectiva del тCT es la innovación y el aprendizaje del personal, seguida de la de clientes, la interna y la financiera. La razón de tomar como primordial a la

\footnotetext{
${ }^{4}$ Utilizar el conocimiento para ser mejor cada día es parte del manejo del CI. Cuando una institución aprende y, con ello crea su propio futuro se torna una "organización inteligente" (Peter Senge, 1990:24). Cuando la empresa transforma el conocimiento en valor se vuelve una empresa del conocimiento (Sullivan, 2001:26).
} 
perspectiva de innovación y aprendizaje se confirma con los resultados de Martín de Castro y López (2008) cuando investigaron el CI en empresas españolas de alta tecnología; estos autores descubrieron que la experiencia y la innovación son los componentes más importantes.

Para extraer conocimiento de los equipos, se pueden emplear las cuatro formas de conversión de conocimiento propuestas por Nonaka y Takeuchi (1995): de tácito a tácito, de tácito a explícito, de explícito a explícito y de explícito a tácito.

La representación gráfica del тст es la siguiente:

Figura 2

Tablero de capacidades tecnológicas (TCT)

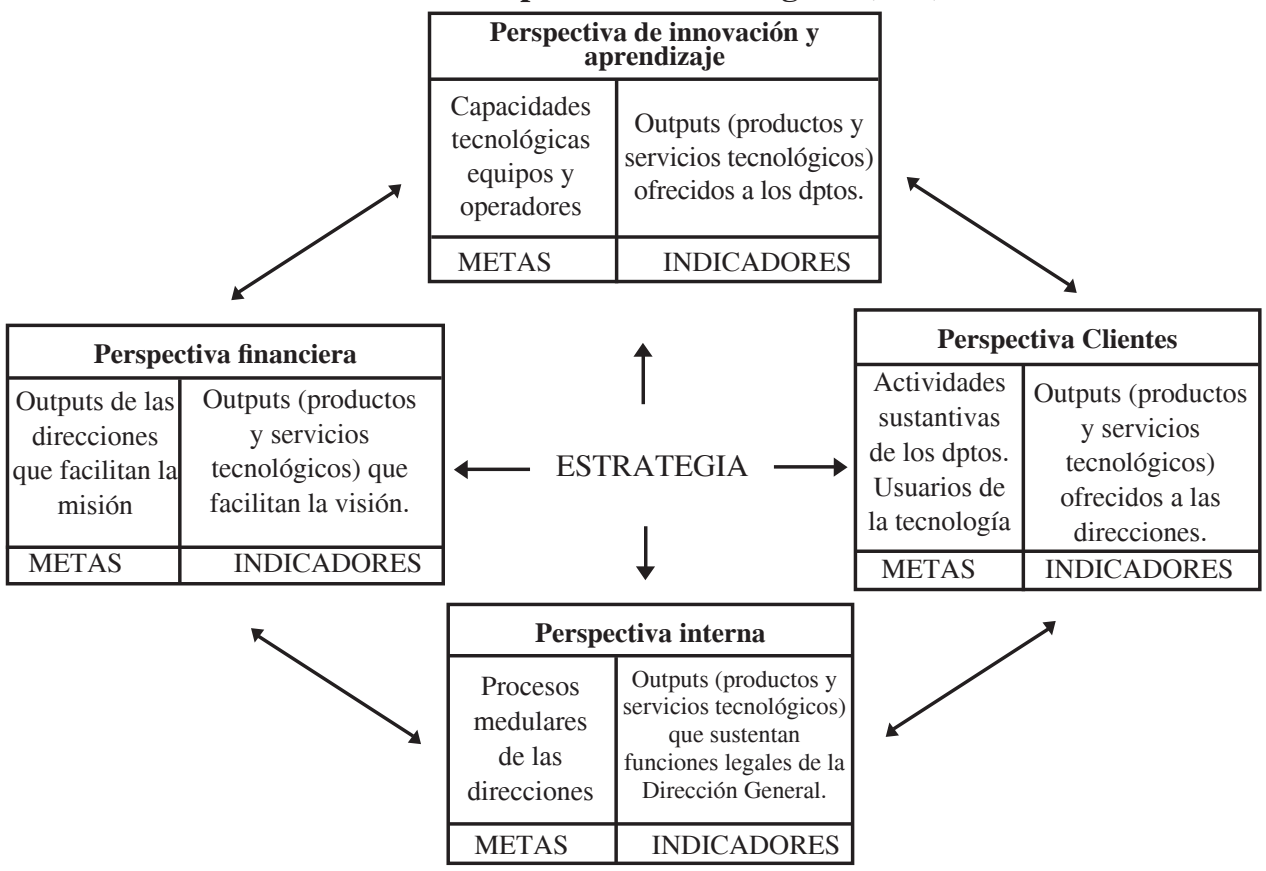

Fuente: elaboración propia con base en Kaplan y Norton (1992).

Las cuatro perspectivas de la figura 2 se vinculan en un flujo: la innovación y aprendizaje lleva a la de clientes; ésta, a la interna; y la interna, a la financiera. También se puede leer de manera inversa porque los procesos medulares de las 
direcciones se nutren de las actividades sustantivas de los departamentos, los empleados y los equipos.

Para operacionalizar el тст se aplica una hoja de control a las direcciones de área usuarias del sIMODI para así medir las metas e indicadores en cada perspectiva, los cuales se anotan en las celdas correspondientes. ${ }^{5}$ Los productos y servicios tecnológicos generados por una perspectiva son los outputs que se transforman en inputs para la siguiente perspectiva. La hoja para operar el тст es:

\section{Cuadro 3}

\section{Hoja de control del tablero de capacidades tecnológicas}

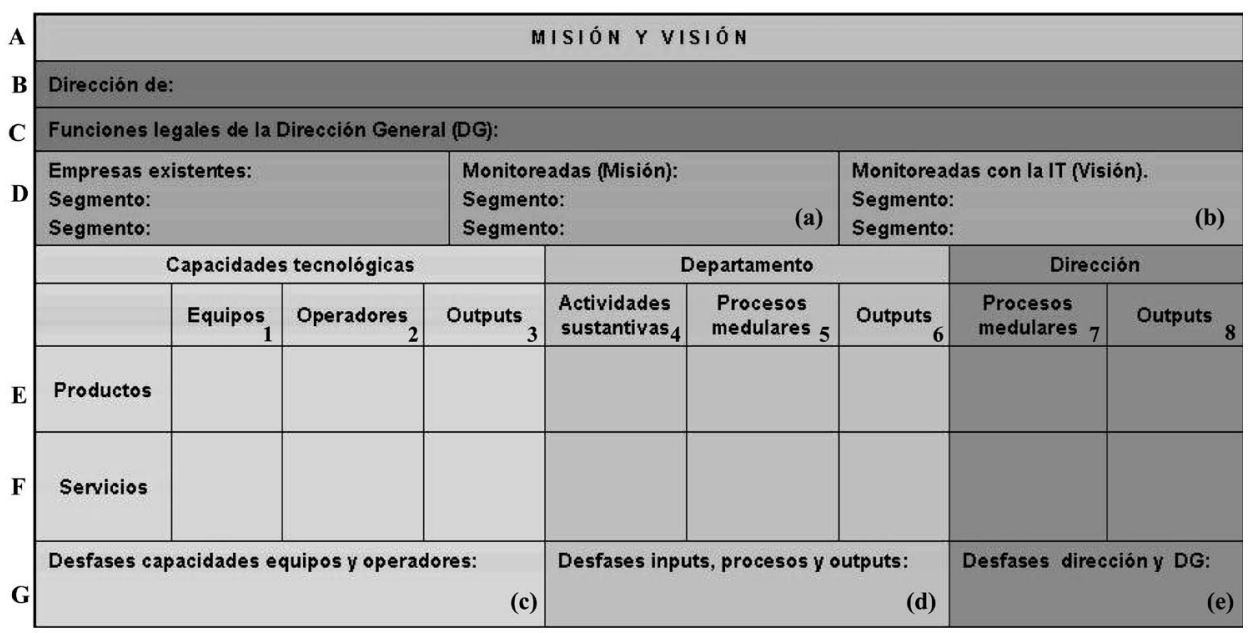

Los outputs de una perspectiva son productos y servicios que se vuelven inputs para la siguiente perspectiva. IT: innovación tecnológica.

La perspectiva de innovación y aprendizaje está compuesta por las capacidades tecnológicas de los operadores y los equipos del simodi; se ubica dentro de las cuatro primeras columnas del lado izquierdo. La meta es determinar las capacidades ofrecidas por los equipos de la IT, y las conocidas y explotadas por los operadores.

\footnotetext{
${ }^{5}$ Para recoger la información sobre las metas e indicadores de las perspectivas se deben analizar manuales (de organización, de procedimientos y de operación, entre otros), fichas técnicas, contratos, leyes, bitácoras de servicios y expedientes, además de aplicar cuestionarios a los operadores del sistema, a los jefes de departamento que integran las direcciones correspondientes, al subdirector de informática y a los directores de área.
} 
El indicador son los outputs generados por la interacción entre ambos elementos, señalados en las celdas E3 y F3. Por ejemplo, en la celda E1 de productos de los equipos se apuntan las prestaciones ofrecidas por los aparatos para generar bienes, y en la E2 las conocidas y explotadas por los operadores del sistema para generar esos bienes, el resultado son los outputs del nivel de aprovechamiento tecnológico que se escriben en la celda E3 (y así sucesivamente con los demás renglones). Con esto se identifican desviaciones entre las capacidades de producción de las máquinas y de los individuos, que se establecen en el renglón G (c); además, da pauta para la adición o eliminación de tareas que no concuerdan. Para eso se requiere modificar los equipos, mejorar la codificación del conocimiento y/o capacitar al personal, es entonces cuando se incrementa el cI.

La perspectiva clientes se refiere a los departamentos usuarios de la tecnología; son clientes internos. La meta es determinar en las celdas E4 y F4 las actividades sustantivas que operan los departamentos con los outputs (productos y servicios) de la perspectiva anterior para desarrollar sus procesos medulares. Los indicadores son los outputs tecnológicos ofrecidos por los departamentos a las áreas, establecidos en las celdas E6 y F6. El flujo de entrada y salida muestra desfases entre los inputs, los procesos y los outputs de los departamentos hacia los procesos medulares de las direcciones. Muestra si los outputs de la perspectiva anterior son los inputs requeridos por esta perspectiva; arroja un balance entre ellas, renglón G (d).

En la perspectiva interna, situada en la columna 7, la meta es establecer en ella los procesos medulares de las direcciones que funcionan con los inputs recibidos de los departamentos (de la columna 6). Los outputs de esta perspectiva son los productos y servicios tecnológicos de la columna 8 que sustentan las funciones legales de la dirección general, anotadas en la fila C; asimismo, muestra la concordancia entre lo producido por el área y las funciones que debe desempeñar la organización de acuerdo con el marco legal, el resultado se asienta en la celda G (e). Una vez más, la hoja de control muestra balances entre las perspectivas, que sirven para corregir desviaciones en su desempeño hasta alcanzar la excelencia (celdas de las filas D y G) con lo cual se enriquece el capital humano y el estructural.

La perspectiva financiera es resultado del flujo tecnológico de las perspectivas anteriores, aparece por su interacción, con ello se incrementa el capital relacional de la organización. La meta es identificar los outputs de la dirección (columna 8), que facilitan a la dirección general el logro de la misión, celda D (a). Los indicadores son el número de empresas nuevas de TV donde la dirección general puede aplicar 
la visión de organismo regulador, celda D (b). El cumplir la misión y la visión arroja mayor cobertura de mercado y, por tanto, mayores ingresos. Así, se cumple la parte financiera del TCT y el requisito comercial de la IT; se obtiene un cambio institucional (OCDE, 1997; Ruttan, 1979).

Una condición para implementar el BSC, según Johanson et al. (2006) es vincular la estructura, los procesos y la creación del conocimiento organizacional; por ello, sugieren relacionar la tecnología con las capacidades del personal y los procesos medulares que desempeñan las áreas para cumplir sus actividades sustantivas con el fin de alcanzar la misión y visión. Todos los elementos van en busca de los mismos objetivos corporativos.

No se pueden alcanzar las metas si uno de ellos carece del conocimiento y los instrumentos necesarios para recibir y transformar los insumos recibidos, así como entregar las salidas esperadas por los usuarios; es decir, cada parte de la organización debe contar con niveles similares de desarrollo para transformar eficientemente los bienes y servicios que entran y salen de sus procesos (Burton \& Pennotti, 2003). Es una cadena de valor tecnológico donde cada perspectiva debe tener estándares técnicos similares para contribuir a la creación de valor a través del flujo. La cadena es la siguiente:

Figura 3

Cadena de valor tecnológico

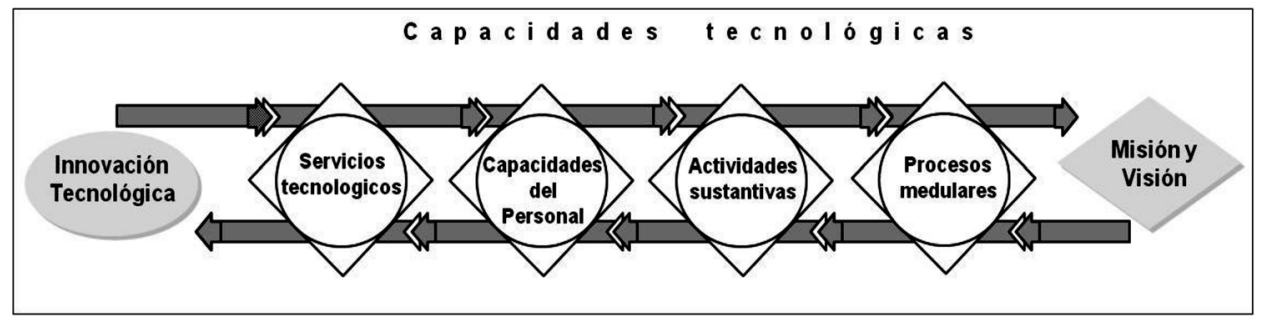

La innovación tecnológica proporciona servicios tecnológicos que soportan las capacidades del personal, los cuales deben estar aplicados en actividades sustantivas orientadas hacia los procesos medulares que permiten alcanzar la misión y visión.

Después de alinear las partes de la cadena de valor tecnológico, se aplica la hoja de control en cada área usuaria del simodi; sus resultados se plasman en cada una de 
las perspectivas del TCT, según corresponda. Para traducir su significado, es necesario que los directivos manejen el lenguaje de los negocios y el de la tecnología (Philip, 2007). Se hace más fácil su comprensión difundiendo entre los integrantes la forma en cómo se diseñaron, organizaron y relacionaron las medidas entre las diversas perspectivas y los objetivos que se persiguen (Lipe \& Salterio, 2002).

\section{Conclusiones}

Utilizar la metodología del Balanced Scorecard para extraer el capital intelectual de la innovación tecnológica permite identificar y administrar los puntos clave donde se relacionan la tecnología y el conocimiento individual y organizacional para alcanzar los objetivos institucionales, además de brindar oportunidades de mejora. Las perspectivas del BSC son flexibles y pueden ser utilizadas por cualquier tipo de sociedad, de ahí que este trabajo se adapte a un proyecto tecnológico de una entidad pública; mediante la creación del TCT se pretende obtener mayor provecho de la tecnología adquirida. El BSC de Kaplan y Norton (1992) incorpora en las acciones estratégicas a todas las áreas de la empresa, excepto a la tecnología, de ahí que se diseñe un método para incluir a esta última en la búsqueda de la misión y visión bajo el enfoque del BSC.

El TCT propuesto proporciona una secuencia lógica del desempeño de la tecnología entre las distintas perspectivas del BSC, lo cual facilita corregir errores en el empleo de recursos, a su vez que permite orientarlos en la búsqueda de la misión o visión. Esto incrementa el ci de la organización y su cobertura de mercado.

El interés principal de toda organización es permanecer vigente en el contexto para lo cual invierte en tecnología. El reto pendiente es extraer el mayor provecho posible de las inversiones en ese rubro, como es el incremento del conocimiento individual y organizacional para crear nuevas capacidades y hacer frente a cambios en el entorno; de manera contraría se subutiliza y sus beneficios se limitan. La inversión no retribuiría la formación de Cr como fuente de creación de valor y de ventaja competitiva $\mathrm{y}$, por tanto, se tendrían que volver a desembolsar recursos en el futuro.

Las diferencias tecnológicas entre las perspectivas y su nivelación originan la creación de CI, al igual que los mecanismos para su interacción y sus salidas al mercado (para concretar la misión y visión). Desde ese panorama, la tecnología y el conocimiento crean un círculo virtuoso donde la tecnología genera mayores conocimientos, a la vez que el conocimiento mejora a la tecnología. 
La IT se subordina al cI y puede ser una plataforma para la reproducción del conocimiento organizacional. Construye las condiciones para conquistar nuevos mercados, siempre y cuando se maneje estratégicamente. De allí la importancia de aplicar de inmediato el тст en las organizaciones. Es uno de los temas pendientes para las empresas públicas o privadas que deseen crecer a partir de la tecnología adquirida.

\section{Referencias}

Arvizu, Ma. del Socorro (2007). El poder WiMax, Recuperado el 14/04/2008, de http://www.elimparcial.com/EdicionImpresa/ejemplaresanteriores/BusquedaEjemplares.asp?numnota $=643298 \&$ fecha $=13 / 8 / 2007$.

Banker, R., H. Chang y M. Pizzini (2004). The Balanced Scorecard: judgmental Effects of Performance Measures Linked to Strategy. The Accounting Review, 79, 1, 1-23.

Betz, F. (1993). Srategic Techbology Management, New York: McGraw-Hill.

Brooking, Annie (1996). El capital intelectual, Barcelona: Paidós.

Burton Herbert, O. y Michael C. Pennotti (2003), The enterprise map: a system for implementing strategy and achieving operational excellence. Engineering Management Journal, sep., 15, 3.

Conacyt (2007). Fondo de Innovación Tecnológica, Secretaría de Economía-Conacyt, Convocatoria 2007-02, México.

Deutschland (2009). La economía creativa. Deutschland. Foro de política, cultura y economía, febrero-marzo, Societäts-Verlag, Berlín.

Diario Oficial de la Federación (2002). "Decreto por el que se autoriza a la SHCP a recibir de los concesionarios de estaciones de radio y TV el pago de impuesto que se indica", México, 10 de octubre.

(2002a), "Reglamento de la Ley Federal de Radio y Televisión, en materia de concesiones, permisos y contenido de las transmisiones de radio y televisión", México, 10 de octubre. 
(2009). “Ley Federal de Radio y Televisión”, México, 19 de mayo.

Edvinsson, L. y M.S. Malone (1997). El capital intelectual, Barcelona: Gestión 2000.

Freeman, C. (1982). The Economics of Industrial Innovation, Cambridge: MA. MIT Press.

García Arrieta, María (1996). Panorámica europea del fondo de comercio, Universidad Complutense de Madrid. Recuperado el 27/07/2006, de http://www. ucm.es/BUCM/cee/doc/0042/03010042.htm.

Gaynor, Gerard H. (1999). Manual de gestión en tecnología, Colombia: McGrawHill Interamericana.

Gestión del conocimiento (2006). Área académica: modelos. Recuperado el 21/08/2006, de http://www.gestiondelconocimiento.com/modelos.htm.

Gómez López, Juan Carlos (2005). El capital intelectual. Recuperado el 25/08/2005, de www.gestiopolis.com/recursos/documentos/fulldocs/ger/capintel.htm

Johanson, Ulf, Chitoshi Koga, Matti Skoog y Johan Henningsson (2006). The Japanese government's intellectual capital reporting guideline, Journal of Intellectual Capital, 7, No. 4, 747-491.

Kaplan, Robert S. y David P. Norton, (1992). El Balanced scorecard: mediciones que impulsan el desempeño. En Harvard Business Review, January- february, 71-79. Reimpresión en español volumen 83, No. 7, julio 2005, 110118.

KPMG (2005). BSC y Dashboard. Oportunidades en servicio de asesoría: Business Intelligence y Balanced Scorecard, México, inteligencia de mercados, abril.

Lev, Baruch (2003). Intangibles, medición, gestión e información, Madrid: Deusto. 
Lipe, M., y S. Salterio (2000). The Judgmental Effects or the Balanced Scorecard's Information Organization and Diversity. The Accounting Review, 75 (3), 283-298.

Lipe, M., y S. Salterio (2002). A note on the Judgmental Effects or the Balanced Scorecard's Information Organization. Accounting Organization and Society, 27, 531-540.

Milena Peñaranda, Constanza (2005). Capital intelectual. Recuperado el 30/08/2005, de http://conymp.blogspot.com/2005/03/capital-intelectual. html.

Martín de Castro, Gregorio y Pedro López Sáez (2008). Intellectual capital in high-tech firms, The case of Spain. Journal of Intellectual Capital, 9, No. 1, 25-36.

Méndez García, Fernando y Fernando Elí Ortiz Hernández (2007, marzo). Capacitar lo invisible. Microempresamexicana, Año 3, No. 37, 34-36.

Murray, Fiona (2004). The role academic inventors in entrepreneurial firms: sharing the laboratory life. Research Policy 33; 643-659.

Narvekar, Rajiv S. y Karuna Jain (2006). A new framework to understand the technological innovation process. Journal of Intellectual Capital, 7, 2, 174186.

Nonaka, Ikujiro y Hirotaka Takeuchi (1995). La organización creadora de conocimiento. Cómo las compañías japonesas crean la dinámica de la innovación, Oxford University Press, $1^{\text {a }}$. Edición en español, 1999.

Norreklit, H. (2000). The Balance on the Balanced Scorecard -a critical analysis of some of its assumptions. Management Accounting Research, 11, 65-88.

oCDE (1997). El manual de Oslo, México: Eurostat, IPN-CIECAS, $1^{\text {a }}$. ed. en español, 2000. 
Pangakar, Ajay and Teresa Kirkwood (2008). Strategic alignment: linking your learning strategy to the balanced scorecard. Industrial and Comercial Training, 40, No. 2, 95-101.

Petty Richard, Federica Ricceri y James Guthrie (2008). Intellectual capital: a user`s perspective, Management Research News, 31, No. 6, 434-447.

Philip, George (2007). IS Strategic Planning for Operational Efficiency. Information Systems Management, Summer, 24, 3, 247-264.

Porter, Michael E. (1982). Estrategia competitiva, México, CECSA, $16^{\mathrm{a}}$ ed. 1992.

Prahalad, C.K. y Hamel Gary (1990), The Core Competence of the Corporation. Harvard Business Review, may-jun, 79-91.

Ruttan, V. (1979). Usher y Schumpeter en la innovación y el cambio tecnológico, en Nathan Rosenberg. Economía del cambio tecnológico, México: FCE, 66-77.

Schumpeter, Joseph A. (1944). Teoría del desenvolvimiento económico, $2^{\mathrm{a}}$ ed. México: FCE, 1997.

Senge, Peter M (1990). La quinta disciplina, México: Granica.

Spivey, W. Austin, J. Michael Munson, William T. Flannery y Fu-Sen Tsai (2009). Improve tech tranfer with this alliance scorecard, Research Technology Management, January-february.

Stewart, Thomas A. (1998). La nueva riqueza de las organizaciones: el capital intelectual, Argentina: Granica.

Sullivan, Patrick H. (2001). Rentabilizar el capital intelectual, técnicas para optimizar el valor de la innovación, Barcelona: Paidós Ibérica.

Sveiby, Karl-Erik (1996). The intangible assets monitor, 15 may, updated december 1997. Recuperado el 29/05/2006, de http://www.sveiby.com/Portals/0/ articles/IntangAss/CompanyMonitor.html. 
(1998). Intellectual Capital and Knowledge Management, 9 april, updated april 2001. Recuperado el 13/03/2006, de http://www.sveiby.com/ articles/IntellectualCapital.html.

Tayles, Mike, Richard H. Pike y Saudan Sofian (2007). Intelectual capital, management acounting practices and corporate performance, perception of managers, Accounting, Auditing \& Accountability Journal, 20, No. 4, 522-548.

Van de Ven A.H. (1986). Central problems in the management of innovation, $M a$ nagement Science, 32, No. 5, 509-607.

Varian Hal, R. (1999). Microeconomía, Un enfoque actual, $5^{\text {a }}$ ed., España: Antoni Bosch editor.

Weinstein, Larry y David Bukovinsky (2009). Use of the Balanced Scorecard and Performance Metrics to achieve operational and strategic alignment in arts and culture not-for profits, International Journal of Arts Management, winter, $42-55$. ( 